\title{
On the distribution \\ of the values of an additive arithmetical function with values in a locally compact abelian group
}

\author{
by \\ JEAN-LOUP MAUCLAIRE (Paris)
}

I. Introduction. Probabilistic number theory deals, among other questions, with the problem of the description of the distribution laws defined by the values of an additive arithmetical function, i.e., by a function $f$ which sends $\mathbb{N}^{*}$ into a group $G$, supposed here additive, satisfying

$$
f(m n)=f(m)+f(n) \quad \text { if }(m, n)=1,
$$

$\mathbb{N}^{*}$ being the set of positive integers.

If $G$ is the additive group of real numbers, denoted by $\mathbb{R}$, a complete result has been given simultaneously by Elliott and Ryavec [6], and Levine and Timofeev [8]. Another case where a complete result has been obtained is when $G$ is the additive group $\mathbb{R} / \mathbb{Z}$, and this case has been considered by many authors, for instance Delange [2], Manstavicius [9], Elliott [5] and Hartman [7]. The case of $G$ discrete has been studied by Ruzsa [13]. In his article [13], Ch. 5, this author sets the following problem for group-valued additive arithmetical functions: "Can one formulate and prove the analogues of the well-known global limit theorems?"

In this article, we consider this problem when the group is a locally compact abelian group.

II. Results. Let $G$ be an additive locally compact abelian group, $g$ its dual group, $m$ a Haar measure on $g$, and $f$ an additive arithmetical function with values in $G$. We denote the integral part of a real number $x$ by $[x]$. If $a$ and $b$ are positive integers, the notation $a \nmid b$ means " $a$ does not divide $b$ ".

$P$ is the set of the prime numbers, $p$ a generic element of $P$, and $E_{x-}$ is the subset of elements $n$ of $\mathbb{N}^{*}$ such that if $p$ divides $n$, then $p \leq x$.

$\delta_{a}$ is the measure with unit mass at the point $a$.

If $H$ is a compact subgroup of $G, T_{H}$ will denote the canonical projection of $G$ on $G / H$. The Haar measures on $g$ and $G$ are normalized as in [11], 
p. 103, and we refer to [1] for generalities on integration on locally compact spaces.

We shall give only one theorem. The interested reader will check that the result given allows one to get, using classical results of probability theory, the well-known formulations in the case of $\mathbb{R}$ or $\mathbb{R} / \mathbb{Z}$, which involve conditions on the values of $f(p), p \in P$, the analogues of Theorems 7.1 and 7.2 in [3] for locally compact abelian groups, of Theorems 8.1, 8.2 and 8.9 in [3] for compact abelian groups, and leads to simple proofs of Theorems 22.1, 22.2 and 22.3 in [4].

THEOREM. Let $h$ be the subset of $g$, in fact a subgroup of $g$, of the elements $\chi$ of $g$ such that

$$
\limsup _{x \rightarrow \infty} \frac{1}{x}\left|\sum_{\substack{n \leq x \\ 2 \nmid n}} \chi(f(n))\right|>0 .
$$

There are two cases:

First case: $h$ is not locally negligible relatively to $m$.

Then the dual of $g / h$ is a compact subgroup $H$ of $G$ and there exists a continuous homomorphism $\varphi: \mathbb{R} \rightarrow G / H$, a sequence $a_{n}$ in $G / H$ and $a$ probability measure $\nu$ with support in $G / H$ such that the sequence of measures

$$
\frac{1}{[x]} \sum_{n \leq x} \delta_{T_{H}(f(n))-\varphi(\log n)-\Sigma_{p \leq x} a_{p}}
$$

converges vaguely to the measure $\nu$, which is not continuous if and only if $H$ is finite and

$$
\sum_{T_{H}(f(p)-\varphi(\log p)) \neq 0} \frac{1}{p}<\infty .
$$

N.B. The sequence $a_{n}$ has the property that for any given $\alpha$ satisfying $0<\alpha \leq 1$ and any sequence $Y_{[x]}$ such that $x^{\alpha} \leq Y_{[x]} \leq x$,

$$
\lim _{x \rightarrow \infty} \sum_{Y_{[x]} \leq p \leq x} a_{p}=0 .
$$

Second case: $h$ is locally negligible relatively to $m$.

In this case, for any compact subgroup $K$ of $G$, any continuous homomorphism $\varphi: \mathbb{R} \rightarrow G / K$, any sequence $a_{n}$ of $G / K$, the sequence of measures

$$
\frac{1}{[x]} \sum_{n \leq x} \delta_{T_{H}(f(n))-\varphi(\log n)-\Sigma_{p \leq x} a_{p}}
$$

converges vaguely to the null measure. 
Acknowledgments. In the first version of this article, only the case of metrizable locally compact abelian groups was considered, and I thank Prof. H. Delange for his remarks concerning the original formulation of the Theorem after its presentation at a meeting on Number Theory held in Oberwolfach in March 1991, which led to the suppression of some ambiguities and to a more precise statement as it appears, for instance, in the announcement of this result in [10], and also Prof. J. Peyrière for helpful discussions. In the present version, the hypothesis of metrizability has been dropped, thanks to Prof. I. Z. Ruzsa who kindly informed me of this possibility if one uses his result in [14]. I thank also the referee for his comments and suggestions, which led, among other things, to a shortening of the paper.

\section{Proof of the result}

\section{A. Preliminaries}

LEMma 1. Let $U$ be an additive locally compact abelian group. If $V$ is a compact neighborhood of the origin of $U$ with characteristic function $I_{V}$, there exists a continuous compactly supported function $I_{1}$ with Fourier transform nonnegative and integrable, hence invertible, such that $I_{1}(0)=1$ and for any $u$ in $U, I_{1}(u) \leq I_{V}(u)$.

Pro of. This is an immediate consequence of Theorem 2.6.2, p. 48 of [12].

LEMMA 2. Let $U$ be an additive locally compact abelian group, and $v$ : $\mathbb{N} \rightarrow U$ a sequence. If for almost every character $\chi$ in $U^{*}$, where $U^{*}$ is the dual of $U$, the sequence $\chi(v(n))$ is convergent, then the sequence $v(n)$ itself is convergent.

Proof. The set $A$ of characters $\chi$ in $U^{*}$ for which the sequence $\chi(v(n))$ is convergent is a subgroup of $U^{*}$, not locally negligible, and so, open and closed, and dense in $U^{*}$. This gives that $A=U^{*}$. Denote by $w(\chi)$ the measurable function defined as the limit of $\chi(v(n))$. If $\chi$ and $\chi^{\prime}$ are in $U^{*}$, we have $w\left(\chi \chi^{\prime}\right)=w(\chi) w\left(\chi^{\prime}\right)$, and the measurability of $w$ implies that $w$ is a character of $U$. So, by the Pontryagin duality theorem, there exists $a$ in $U$ such that for every $\chi$ in $U^{*}, \chi(v(n)-a)$ tends to 1 . This implies that $v(n)-a$ tends to 0 .

HALÁSZ'S THEOREM. Let $g(n), n=1,2, \ldots$, be a multiplicative function with modulus equal to 1 . If there exists a real number $u$ such that $\sum p^{-1}(1-$ $\left.\operatorname{Re} g(p) p^{-i u}\right)$ converges, then

$$
\limsup _{x \rightarrow \infty} \frac{1}{x}\left|\sum_{\substack{n \leq x \\ 2 \nmid n}} g(n)\right|>0
$$


and

$$
\sum_{n \leq x} g(n)=\frac{x^{1+i u}}{1+i u} \prod_{p \leq x}\left(1-\frac{1}{p}\right)\left(1+\sum_{r=1}^{\infty} \frac{g\left(p^{r}\right) p^{-i u r}}{p^{r}}\right)+o(x), \quad x \rightarrow \infty .
$$

If such a $u$ does not exist, then

$$
\lim _{x \rightarrow \infty} \frac{1}{x}\left|\sum_{n \leq x} g(n)\right|=0 .
$$

(See [3], Ch. 6.)

RUzSA's TheOREM. Let $G$ be a locally compact abelian group, $\mu_{p}$ a sequence of probability measures on $G$, and denote by $\mu_{y-}$ the convolution product

$$
\mu_{y-}=\underset{p \leq y}{*} \mu_{p} .
$$

If there exists $V$, a compact neighborhood of the origin in $G$, such that

$$
\lim _{y \rightarrow \infty} \sup _{x \in G} \mu_{y-}(x+V)>0,
$$

then there exists a sequence $a_{y-}$ in $G$ and a probability measure $\nu$ such that the sequence of measures $\mu_{y-} * \delta_{-a_{y-}}$ converges vaguely to the measure $\nu$.

(See [14].)

B. Main proposition. The basic result is

Proposition 1. The following assertions are equivalent:

(1) $h$ is not locally negligible,

(2) there exists a continuous homomorphism $\varphi: \mathbb{R} \rightarrow G / H$, where $H$ is a compact group, such that for every $\chi$ in $h$, the dual of $G / H$, we have

$$
\lim _{x \rightarrow \infty}\left|\prod_{\substack{p \leq x \\ p \neq 2}}\left(1-\frac{1}{p}\right) \sum_{k \geq 0} \frac{\chi\left(f\left(p^{k}\right)-\varphi\left(\log p^{k}\right)\right)}{p^{k}}\right|>0 .
$$

Proof. $(1) \Rightarrow(2)$. We assume that $h$ is not locally negligible.

$\mathrm{P} 1 . h$ is an open subgroup of $g$.

Pro of of P1. $h$ is the set defined by

$$
h=\left\{\chi \in g: \limsup _{x \rightarrow \infty} \frac{1}{x}\left|\sum_{\substack{n \leq x \\ 2 \nmid n}} \chi(f(n))\right|>0\right\} .
$$

$\chi(f(n))$ is multiplicative with modulus equal to 1 , and so, Halász's Theorem gives that $\chi \in h$ if and only if there exists $\tau_{\chi} \in \mathbb{R}$ such that

$$
\sum \frac{1-\operatorname{Re} \chi(f(p)) p^{-i \tau_{\chi}}}{p} \text { converges. }
$$


Since for every $\chi$ and $\chi^{\prime}$ in $g$ and $a$ in $G$, we have

$$
\left|1-\chi \chi^{\prime}(a)\right|^{2} \leq 2\left(|1-\chi(a)|^{2}+\left|1-\chi^{\prime}(a)\right|^{2}\right),
$$

we see that if $\sum\left(1-\operatorname{Re} \chi(f(p)) p^{-i \tau_{\chi}}\right) / p$ and $\sum\left(1-\operatorname{Re} \chi^{\prime}(f(p)) p^{\left.-i \tau_{\chi^{\prime}}\right) / p}\right.$ converge, then so does $\sum\left(1-\operatorname{Re} \chi \chi^{\prime}(f(p)) p^{-i\left(\tau_{\chi}+\tau_{\chi^{\prime}}\right)}\right) / p$, and so, $\chi \chi^{\prime}$ belongs to $h . h$ is clearly measurable, and being a not locally negligible measurable subgroup of $g, h$ is an open subgroup of $g$; hence, it is closed, and so, locally compact; moreover, $g / h$ is discrete. We denote by $H$ the orthogonal of $h$, which is compact as the dual group of a discrete group. A consequence is that $G / H$ is locally compact.

P2. $\chi \rightarrow \tau_{\chi}$ is a group homomorphism.

Proof of P2. We prove that $\chi \rightarrow \tau_{\chi}$ is a function $h \rightarrow \mathbb{R}$ and that $\tau_{\chi \chi^{\prime}}=\tau_{\chi}+\tau_{\chi^{\prime}}$. that

Suppose that $\tau_{\chi}$ and $\tau_{\chi}^{\prime}$ are associated with the same $\chi$ of $h$. This gives

$$
\sum_{p} \frac{1-\operatorname{Re} \chi \bar{\chi}(f(p)) p^{-i\left(\tau_{\chi}-\tau_{\chi}^{\prime}\right)}}{p} \text { converges, }
$$

i.e. $\sum\left(1-\operatorname{Re} p^{-i\left(\tau_{\chi}-\tau_{\chi}^{\prime}\right)}\right) / p$ converges, which implies $\tau_{\chi}=\tau_{\chi}^{\prime}$.

To show that $\tau_{\chi \chi^{\prime}}=\tau_{\chi}+\tau_{\chi^{\prime}}$, we remark that $\sum\left(1-\operatorname{Re} \chi(f(p)) p^{-i \tau_{\chi}}\right) / p$ and $\sum\left(1-\operatorname{Re} \chi^{\prime}(f(p)) p^{\left.-i \tau_{\chi^{\prime}}\right) / p}\right.$ converge, and so, as above, we conclude that $\sum\left(1-\operatorname{Re} \chi \chi^{\prime}(f(p)) p^{-i\left(\tau_{\chi}+\tau_{\chi^{\prime}}\right)}\right) / p$ converges.

P3. $\chi \rightarrow \tau_{\chi}$ is a continuous group homomorphism.

Proof of P3. It is sufficient to show that $\chi \rightarrow \tau_{\chi}$ is measurable.

For any prime $q$, we define, for $\alpha>0, \alpha$ an integer, and $\chi$ in $h$,

$$
S_{q^{\alpha}, x}^{1}(\chi)=\sum_{\substack{n \leq x \\ 2 q^{\alpha} \nmid n}} \chi(f(n)) .
$$

These functions are continuous on $h$, and setting

$$
S_{q^{\alpha}, x}(\chi)=S_{q^{\alpha}, x}^{1}(\chi)+I\left(S_{q^{\alpha}, x}^{1}(\chi)\right),
$$

where $I(z)$ is defined by $I(z)=1$ if $z=0, I(z)=0$ if $z \neq 0$, we see that $S_{q^{\alpha}, x}(\chi)$ is a sequence of measurable functions, never equal to 0 , and we have

$$
\frac{S_{q^{2}, x}(\chi)}{S_{q^{1}, x}(\chi)} \rightarrow 1+\frac{\chi(f(q)) q^{-i \tau_{\chi}}}{q}, \quad x \rightarrow \infty .
$$

So, as a limit of a sequence of measurable functions, $q^{-i \tau_{\chi}}$ is measurable. 
Now, since

$$
\begin{aligned}
{\left[\sum_{\substack{n \leq q \\
2 \nmid n}} \chi(f(n))\right]\left[q^{1+i \tau_{\chi}} \prod_{p \leq q, p \neq 2}\left(1-\frac{1}{p}\right)\right.} & \left.\left(\sum_{k \geq 0} \frac{\chi\left(f\left(p^{k}\right)\right) p^{-i k \tau_{\chi}}}{p^{k}}\right)\right]^{-1} \\
& \rightarrow \frac{1}{1+i \tau_{\chi}}, \quad q \text { prime, } q \rightarrow \infty,
\end{aligned}
$$

we deduce that $\chi \rightarrow \tau_{\chi}$ is measurable as the limit of a sequence of measurable functions.

P4. There exists a continuous homomorphism $\varphi: \mathbb{R} \rightarrow G / H$ such that for every $n$ in $\mathbb{N}^{*}$, we have $\chi(\varphi(\log n))=\exp \left\{i \tau_{\chi} \log n\right\}$.

Proof of P4. For $a$ in $\mathbb{R}$, we set $\psi_{a}(\chi)=\exp \left\{i a \tau_{\chi}\right\}$, and remark that $\chi \rightarrow \psi_{a}(\chi)$ is a continuous character of $h$. The classical duality theory (see [15], Ch. VI, $\S 28$, pp. 102-103) gives immediately that the dual map $\varphi$ : $\mathbb{R} \rightarrow G / H$ defined by $\chi(\varphi(a))=\exp \left\{i \tau_{\chi} a\right\}$ is a continuous homomorphism $\mathbb{R} \rightarrow G / H$.

This ends the proof of $(1) \Rightarrow(2)$.

$(2) \Rightarrow(1)$. We assume that there exists a continuous homomorphism $\varphi$ : $\mathbb{R} \rightarrow G / H$, where $H$ is a compact group, such that for every $\chi$ in $h$, the dual of $G / H$, we have

$$
\lim _{x \rightarrow \infty}\left|\prod_{\substack{p \leq x \\ p \neq 2}}\left(1-\frac{1}{p}\right) \sum_{k \geq 0} \frac{\chi\left(f\left(p^{k}\right)-\varphi\left(\log p^{k}\right)\right)}{p^{k}}\right|>0 .
$$

Since such a continuous homomorphism $\varphi: \mathbb{R} \rightarrow G / H$ exists, it follows that for every $\chi$ in $h, \chi(\varphi(a))$ is a character of $\mathbb{R}$, and so, there exists $\tau_{\chi}$ such that $\chi(\varphi(a))=\exp \left\{i \tau_{\chi} a\right\}$. Halász's Theorem gives immediately that

$$
\sum_{\substack{n \leq x \\ 2 \nmid n}} \chi(f(n))=\frac{x^{1+i \tau_{\chi}}}{1+i \tau_{\chi}} \prod_{\substack{p \leq x \\ p \neq 2}}\left(1-\frac{1}{p}\right) \sum_{k \geq 0} \frac{\chi\left(f\left(p^{k}\right)\right) p^{-i k \tau_{\chi}}}{p^{k}}+o(x), \quad x \rightarrow \infty,
$$

and this shows that for every $\chi$ in $h$, which is open since $g / h$ is discrete, and so, not locally negligible, we have

$$
\limsup _{x \rightarrow \infty} \frac{1}{x}\left|\sum_{\substack{n \leq x \\ 2 \nmid n}} \chi(f(n))\right|>0 .
$$

This ends the proof of Proposition 1.

C. Proof of the Theorem.

Proof of the first case. 1 . Since $h$ is not locally negligible, by Proposition 1, there exists a compact group $H$ such that $h$ is the dual of 
$G / H$ and a continuous homomorphism $\varphi: \mathbb{R} \rightarrow G / H$ such that for every $\chi$ in $h$, we have

$$
\lim _{x \rightarrow \infty}\left|\prod_{\substack{p \leq x \\ p \neq 2}}\left(1-\frac{1}{p}\right) \sum_{k \geq 0} \frac{\chi\left(f\left(p^{k}\right)-\varphi\left(\log p^{k}\right)\right)}{p^{k}}\right|>0 .
$$

We define

$$
\begin{aligned}
& \widetilde{\mu}_{y-}=\prod_{p \leq x}\left(1-\frac{1}{p}\right) \sum_{n \in E_{x-}} \frac{\delta_{T_{H}(f(n))-\varphi(\log n)}}{n}, \\
& \widetilde{\mu}_{y-}^{s}=\prod_{p \leq x}\left(1-\frac{1}{p}\right)^{2} \sum_{(m, n) \in E_{x-}^{2}} \frac{\delta_{T_{H}(f(m)-f(n))-\varphi(\log m-\log n)}}{m n}
\end{aligned}
$$

and we consider a compact symmetric neighborhood $V$ of the origin in $G / H$. Applying Lemma 1 with $U=G / H$, we can find a continuous compactly supported function $I_{1}$ with Fourier transform $F\left(I_{1}(\chi)\right)$ nonnegative and integrable, hence invertible, such that $I_{1}(0)=1$ and for any $u$ in $G / H$, $I_{1} \leq I_{V}$. We have

$$
\widetilde{\mu}_{y-}^{s}(V)=\int I_{V} d \widetilde{\mu}_{y-}^{s} \geq \int I_{1} d \widetilde{\mu}_{y-}^{s},
$$

since $I_{1} \leq I_{V}$, and also

$$
\int I_{1} d \widetilde{\mu}_{y-}^{s}=\int_{h} F\left(I_{1}(\chi)\right)\left|\prod_{p \leq y}\left(1-\frac{1}{p}\right)\left(1+\sum_{r \geq 1} \frac{\chi\left(f\left(p^{r}\right)\right) p^{-r i \tau_{\chi}}}{p^{r}}\right)\right|^{2} d m(\chi) .
$$

Now, we remark that

$$
\begin{aligned}
& \lim _{x \rightarrow \infty} \int_{h} F\left(I_{1}(\chi)\right) \mid\left.\prod_{p \leq x}\left(1-\frac{1}{p}\right)\left(1+\sum_{r \geq 1} \frac{\chi\left(f\left(p^{r}\right)\right) p^{-r i \tau_{\chi}}}{p^{r}}\right)\right|^{2} d m(\chi) \\
&=\int_{h} F\left(I_{1}(\chi)\right) \lim _{x \rightarrow \infty}\left|\prod_{p \leq x}\left(1-\frac{1}{p}\right)\left(1+\sum_{r \geq 1} \frac{\chi\left(f\left(p^{r}\right)\right) p^{-r i \tau_{\chi}}}{p^{r}}\right)\right|^{2} d m(\chi)
\end{aligned}
$$

by the Lebesgue theorem, and since the integrand is a continuous function taking a positive value at the origin, this integral is positive, has a positive value $c$, and for $x$ large enough, is greater than $c / 2$. This gives that

$$
\lim _{y \rightarrow \infty} \widetilde{\mu}_{y-}^{s}(V)>0 \text {. }
$$

Since this inequality can be written as

$$
\lim _{y \rightarrow \infty} \int_{G / H} \widetilde{\mu}_{y-}(x+V) d \mu_{y-}(x)>0,
$$


this implies that

$$
\lim _{y \rightarrow \infty} \sup _{x \in G / H} \widetilde{\mu}_{y-}(x+V)>0 .
$$

Now, by Ruzsa's Theorem, there exists a sequence $a_{n}$ in $G / H$ and a probability measure $\nu$ with support in $G / H$ such that the sequence of measures

$$
\mu_{y-}=\prod_{p \leq x}\left(1-\frac{1}{p}\right) \sum_{n \in E_{x-}} \frac{\delta_{T_{H}(f(n))-\varphi(\log n)-\Sigma_{p \leq x} a_{p}}}{n}
$$

converges vaguely to the measure $\nu$.

2. Pro of of the N.B. We have to prove that the sequence $a_{n}$ has the property that for any given $\alpha$ satisfying $0<\alpha \leq 1$ and any sequence $Y_{[x]}$ such that $x^{\alpha} \leq Y_{[x]} \leq x$,

$$
\lim _{x \rightarrow \infty} \sum_{Y_{[x]} \leq p \leq x} a_{p}=0 .
$$

Let $F(n)=T_{H}(f(n))-\varphi(\log n)$. We remark that for $\chi$ in $h$,

$$
\bar{\chi}\left(\sum_{\substack{p \leq y \\ p \neq 2}} a_{p}\right) \prod_{\substack{p \leq y \\ p \neq 2}}\left(1-\frac{1}{p}\right) \sum_{k \geq 0} \frac{\chi\left(F\left(p^{k}\right)\right)}{p^{k}}
$$

converges and the limit is not 0 . By the Cauchy criterion, we have, for any given $\alpha$ satisfying $0<\alpha \leq 1$ and any sequence $Y_{[x]}$ such that $x^{\alpha} \leq Y_{[x]} \leq x$,

$$
\lim _{x \rightarrow \infty} \bar{\chi}\left(\sum_{Y_{[x]} \leq p \leq x} a_{p}\right) \prod_{Y_{[x]} \leq p \leq x}\left(1-\frac{1}{p}\right) \sum_{k \geq 0} \frac{\chi\left(F\left(p^{k}\right)\right)}{p^{k}}=1 .
$$

Now, since

$$
\left|\prod_{p \leq x}\left(1-\frac{1}{p}\right) \sum_{k \geq 0} \frac{\chi\left(F\left(p^{k}\right)\right)}{p^{k}}\right| \quad \text { converges }
$$

we have

$$
\lim _{x \rightarrow \infty} \log \left|\prod_{Y_{[x]} \leq p \leq x}\left(1-\frac{1}{p}\right) \sum_{k \geq 0} \frac{\chi\left(F\left(p^{k}\right)\right)}{p^{k}}\right|=0,
$$

which implies

$$
\lim _{x \rightarrow \infty} \sum_{Y_{[x]} \leq p \leq x} \frac{1-\operatorname{Re} \chi(F(p))}{p}=0,
$$

which can be written as

$$
\lim _{x \rightarrow \infty} \sum_{Y_{[x]} \leq p \leq x} \frac{|1-\chi(F(p))|^{2}}{p}=0 .
$$


But

$$
\log \prod_{Y_{[x]} \leq p \leq x}\left(1-\frac{1}{p}\right) \sum_{k \geq 0} \frac{\chi\left(F\left(p^{k}\right)\right)}{p^{k}}=\sum_{Y_{[x]} \leq p \leq x} \frac{1-\chi(F(p))}{p}+o(1),
$$

and

$$
\begin{aligned}
\left|\sum_{Y_{[x]} \leq p \leq x} \frac{1-\chi(F(p))}{p}\right| & \leq \sum_{Y_{[x]} \leq p \leq x} \frac{|1-\chi(F(p))|}{p} \\
& \leq\left(\sum_{Y_{[x]} \leq p \leq x} \frac{|1-\chi(F(p))|^{2}}{p}\right)^{1 / 2}\left(\sum_{Y_{[x]} \leq p \leq x} \frac{1}{p}\right)^{1 / 2} \\
& \leq\left(\sum_{x^{\alpha} \leq p \leq x} \frac{|1-\chi(F(p))|^{2}}{p}\right)^{1 / 2}\left(\sum_{x^{\alpha} \leq p \leq x} \frac{1}{p}\right)^{1 / 2},
\end{aligned}
$$

and since

$$
\left(\sum_{x^{\alpha} \leq p \leq x} \frac{1}{p}\right)^{1 / 2}=O(1)
$$

and

we get

$$
\left(\sum_{x^{\alpha} \leq p \leq x} \frac{|1-\chi(F(p))|^{2}}{p}\right)^{1 / 2}=o(1), \quad x \rightarrow \infty,
$$

which implies

$$
\lim _{x \rightarrow \infty} \log \prod_{Y_{[x]} \leq p \leq x}\left(1-\frac{1}{p}\right) \sum_{k \geq 0} \frac{\chi\left(F\left(p^{k}\right)\right)}{p^{k}}=0,
$$

From

$$
\lim _{x \rightarrow \infty} \prod_{Y_{[x]} \leq p \leq x}\left(1-\frac{1}{p}\right) \sum_{k \geq 0} \frac{\chi\left(F\left(p^{k}\right)\right)}{p^{k}}=1 .
$$

$$
\lim _{x \rightarrow \infty} \bar{\chi}\left(\sum_{Y_{[x]} \leq p \leq x} a_{p}\right) \prod_{Y_{[x]} \leq p \leq x}\left(1-\frac{1}{p}\right) \sum_{k \geq 0} \frac{\chi\left(F\left(p^{k}\right)\right)}{p^{k}}=1,
$$

we deduce that

$$
\lim _{x \rightarrow \infty} \chi\left(\sum_{Y_{[x]} \leq p \leq x} a_{p}\right)=1
$$

and this implies by Lemma 2 that $L=\lim _{x \rightarrow \infty} \sum_{Y_{[x]} \leq p \leq x} a_{p}$ exists, and $L=0$.

3. Continuity of $\nu$. We prove the equivalence of

(1) $\nu$ is not continuous,

(2) $H$ is finite and $\sum_{T_{H}(f(p)-\varphi(\log p)) \neq 0} 1 / p<\infty$. 
Proof. Since $H$ and the sequence of measures

$$
\mu_{y-}=\prod_{p \leq x}\left(1-\frac{1}{p}\right) \sum_{n \in E_{x-}} \frac{\delta_{T_{H}(f(n))-\varphi(\log n)-\Sigma_{p \leq x} a_{p}}}{n}
$$

which can be written as

$$
\mu_{y-}=\underset{p \leq x}{*}\left(1-\frac{1}{p}\right) \sum_{k=0}^{\infty} \frac{\delta_{T_{H}\left(f\left(p^{k}\right)\right)-\varphi\left(\log p^{k}\right)-a_{p}}}{p^{k}},
$$

satisfy the hypothesis of Theorem 3.2 of [7], the measure $\nu$ is not continuous if and only if $H$ is finite and

$$
\lim _{y \rightarrow \infty} \prod_{p \leq y} S_{p} \neq 0
$$

where $S_{p}$ is defined by

$$
S_{p}=\max _{u \in G / H}\left(\left(1-\frac{1}{p}\right) \sum_{k=0}^{\infty} \frac{\delta_{T_{H}\left(f\left(p^{k}\right)\right)-\varphi\left(\log p^{k}\right)-a_{p}}}{p^{k}}\right)(u) .
$$

This is equivalent to $\sum_{p \in P}\left(1-S_{p}\right)<\infty$ which is true if and only if

$$
\sum_{p \in P}\left(1-\max _{u \in G / H}\left(\left(1-\frac{1}{p}\right) \delta_{-a_{p}}+\frac{\delta_{T_{H}(f(p))-\varphi(\log p)-a_{p}}}{p}\right)(u)\right)<\infty .
$$

It is clear that this inequality means that for some sequence $\left(u_{p}\right), p \in P$, we have

$$
\sum_{p \in P}\left(1-\delta_{-a_{p}}\left(u_{p}\right)\right)<\infty
$$

and

$$
\sum_{p \in P} \frac{\delta_{-a_{p}}\left(u_{p}\right)-\delta_{T_{H}\left(f(p)-\varphi(\log p)-a_{p}\right)}\left(u_{p}\right)}{p}<\infty .
$$

It is easy to check that these inequalities can be satisfied if and only if the condition

is satisfied.

$$
\sum_{T_{H}(f(p)-\varphi(\log p)) \neq 0} \frac{1}{p}<\infty
$$

Proof of the second case. If $m(h)=0$, we have

$$
\lim _{x \rightarrow \infty}\left|\frac{1}{x} \sum_{n \leq x} \chi(f(n))\right|=0 \quad d m \text {-a.e. }
$$

If $K$ is a compact subgroup of $G$ and $\varphi$ is a continuous homomorphism from $\mathbb{R}$ to $G / K$, the dual group $k$ of $G / K$ is open and the duality theory gives 
that for every $\chi$ in $k$, there exists a real number $\tau_{\chi}$ such that for every $t$ in $\mathbb{R}^{*}$, we have

$$
\chi(\varphi(t))=\exp \left\{i \tau_{\chi} t\right\}
$$

which gives that for every $n$ in $\mathbb{N}^{*}$,

$$
\chi(\varphi(\log n))=\exp \left\{i \tau_{\chi} \log n\right\} .
$$

Since $m(h)=0$, by Halász's Theorem, we know that for every real $u$, we have $d m$-a.e.

$$
\left|\sum_{n \leq x} \chi(f(n)) n^{i u}\right|=o(x), \quad x \rightarrow \infty .
$$

This implies that for almost every $\chi$ in $k$,

$$
\left|\sum_{n \leq x} \chi(f(n)) n^{-i \tau_{\chi}}\right|=o(x), \quad x \rightarrow \infty,
$$

and so, for almost every $\chi$ in $k$,

$$
\left|\sum_{n \leq x} \chi(f(n)-\varphi(\log n))\right|=o(x), \quad x \rightarrow \infty,
$$

which implies immediately that whatever the sequence $a_{n}$ is, we have

$$
\lim _{x \rightarrow \infty} \frac{1}{x} \sum_{n \leq x} \chi\left(f(n)-\varphi(\log n)-\sum_{p \leq x} a_{p}\right)=0,
$$

for almost every $\chi$ in $k$, and this ends the proof of the second case of the Theorem.

\section{References}

[1] N. Bourbaki, Eléments de mathématique. Intégration, chap. I-IV, 2ème éd., Hermann, Paris, 1965.

[2] H. Delange, On the distribution modulo 1 of additive functions, J. Indian Math. Soc. 34 (1970), 215-235.

[3] P. D. T. A. Elliott, Probabilistic Number Theory I, Grundlehren Math. Wiss. 239, Springer, New York, 1979.

[4] - , Arithmetic Functions and Integer Products, Grundlehren Math. Wiss. 272, Springer, New York, 1985.

[5] - On the limiting distribution of additive functions $(\bmod 1)$, Pacific J. Math. 38 (1971), 49-59.

[6] P. D. T. A. Elliott and C. Ryavec, The distribution of values of additive arithmetical functions, Acta Math. 126 (1971), 143-164.

[7] P. Hartman, Infinite convolutions on locally compact abelian groups and additive functions, Trans. Amer. Math. Soc. 214 (1975), 215-231.

[8] B. V. Levine and N. M. Timofeev, On the distribution of values of additive functions, Acta Arith. 26 (1975), 333-364. 
[9] E. Manstavicius, The distribution of additive arithmetic functions modulo 1, Litovsk. Mat. Sb. 13 (1973), 101-108.

[10] J. L. Mauclaire, Distribution des valeurs d'une fonction arithmétique additive à valeurs dans un groupe abélien localement compact métrisable, C. R. Acad. Sci. Paris Sér. I 313 (1991), 345-348.

[11] H. Reiter, Classical Harmonic Analysis and Locally Compact Groups, Clarendon Press, Oxford, 1968.

[12] W. Rudin, Fourier Analysis on Groups, 2nd ed., Interscience Publishers, New York, 1967.

[13] I. Z. Ruzsa, General multiplicative functions, Acta Arith. 32 (1977), 313-347.

[14] - Infinite convolution and shift-convergence of measures on topological groups, in: Probability Measures on Groups (Proc. Conf., Oberwolfach 1981), Lecture Notes in Math. 928, Springer, New York, 1982, 337-353.

[15] A. Weil, L'intégration dans les groupes topologiques et ses applications, 2ème éd., Hermann, Paris, 1965.

C.N.R.S., U.R.A. 212

THÉORIES GÉOMÉTRIQUES

UNIVERSITÉ PARIS-VII

TOUR $\mathrm{N}^{\circ} 45-55,5^{\mathrm{e}}$ ÉTAGE

2, PLACE JUSSIEU

75251 PARIS CEDEX 05

FRANCE

Received on 12.10.1992

and in revised form on 14.12.1993 